\title{
Synthesis and Structural, Optical Properties of Cadmium Doped Cobalt Oxide Nanoparticles
}

\author{
M. Mayakannan ${ }^{1,}{ }^{*}$, E. Vinoth ${ }^{1}$, S. Prabakar ${ }^{2}$ \\ ${ }^{1}$ PG \& Research Department of Physics, Government Arts College, Thiruvalluvar University, Tiruvannamalai-606603, Tamil Nadu, \\ India. \\ 2 Department of Physics, Siga college of Management and Computer Science, Thiruvalluvar University, Villupuram-605601, Tamil \\ Nadu, India \\ ${ }^{\star}$ Corresponding author email: mmayakannan92@gmail.com \\ DOI: https://doi.org/10.34256/irjmt2135
}

Received: 10-03-2021, Revised: 07-05-2021, Accepted: 08-05-2021, Published: 11-05-2021

\begin{abstract}
The nanostructure cadmium doped cobalt oxide nanoparticles were prepared by microwave irradiation techniques. The prepared nanoparticles were further characterized using Powder X-ray diffraction, Fourier transform infrared spectroscopy and UV-Vis spectroscopy. The Powder $X$ ray diffraction results shows good crystalline nature. The Fourier transform infrared spectroscopy conforms stretching and bending vibration of metal oxygen groups. UVVis spectroscopy results show absorption edges are $204 \mathrm{~nm}, 220 \mathrm{~nm}$ prepared nanoparticles additionally optical parameter skin depth, extinction co-efficient, reflectance, refractive index is calculated.
\end{abstract}

Keywords: Nanoparticles, Microwave-assisted, Functional groups, Optical properties

\section{Introduction}

In recent years, Nanoparticles vital role in engineering, medicine, chemistry, physics etc. The attenuation has made to synthesis nanoparticles (NPs) based on their size; shape is [1-2]. In this view, cobalt oxide nanoparticles consider to be super capacitor, lithium-ion batteries, gas sensor application were reported [3-10].

The synthesis of cobalt oxide NPs by sol gel, thermal, son chemical and precipitation methods [1118]. Recently, Metal ions doped cobalt oxide nanoparticles physical and chemical properties are varied. Also, cadmium doped $\mathrm{Co} 3 \mathrm{O} 4$ nanosheets prepared electrode materials [19] $\mathrm{Cd}$ doped CoS nanocluster alloys [20]. Microwave heating methods synthesis of NPs due to the high purity, shorter reaction time, low cost by internal volumetric heating process [2124]. In this present research work, the attempt was fall on to synthesis of cadmium doped cobalt oxide nanoparticles by precipitation methods. The optical parameter skin depth, extinction co-efficient, reflectance, refractive index is calculated for analysis of optical properties.

\section{Experimental details}

2.1 Materials and methods

$\mathrm{Cd}-\mathrm{Co}_{3} \mathrm{O}_{4} \mathrm{NPs}$ were synthesized by taking cobalt (II) nitrates hexahydrate (AR grade $99 \%$ pure) and cadmium sulphate (AR grade $99 \%$ pure) and $25 \%$ ammonia solution (Merck) chemicals are used. All other chemicals used were of reagent grade and double distilled water used as a solvent.

A $0.2 \mathrm{M}$ concentration solution of cobalt nitrate hexahydrate was prepared by dissolving in double distilled water. Then cadmium sulphate of 5 weight percentage was added to the cobalt hydroxyl solution.

$\mathrm{pH}$ value of the solution was maintained at 8 precipitation were obtained. The sample dried hot air oven at $100^{\circ} \mathrm{C}$ for 2 hours and the prepared sample was annealed up to temperature of $600{ }^{\circ} \mathrm{C}$ for 2 hours collected sample A. The similar way prepared samples precipitation was irradiated by microwave radiation frequency $2.45 \mathrm{GHz}$ and power up to $1 \mathrm{KW}$ for 5 minutes continuously. The prepared sample was annealed at a temperature of $600^{\circ} \mathrm{C}$ for 2 hours collected sample B.

\subsection{Characterization}

The structural properties of the samples were analyzed by Powder X-Ray Diffraction using a Bruker AXS D8 Advance instrument and the monochromatic wavelength of the $1.5406 \AA$ over the diffraction angle range of $2 \theta 10^{\circ}-100^{\circ}$. The functional group of the sample identified using Attenuated total reflectance 
spectroscopy was recorded by a carry 50 ATR- FTIR spectrometer, The Ultraviolet spectrum was analysis carry 60 Uv-Vis Spectro photometers

\section{Results and discussion}

\subsection{Powder X-ray diffraction}

The phase of the as-prepared samples cadmium doped $\mathrm{Co}_{3} \mathrm{O}_{4}$ was investigated by Powder $\mathrm{X}$ ray diffraction. Figure.1. (A), (B) Shows the diffraction peak can be assigned to $31^{\circ}, 36^{\circ}, 59^{\circ}, 65^{\circ}$ indexed as (220), (311), (511), (440) lattice planes of cobalt oxide (JCPDS NO 76-1802).

\subsection{Attenuated total reflectance (ATR)}

The various functional groups presented in the

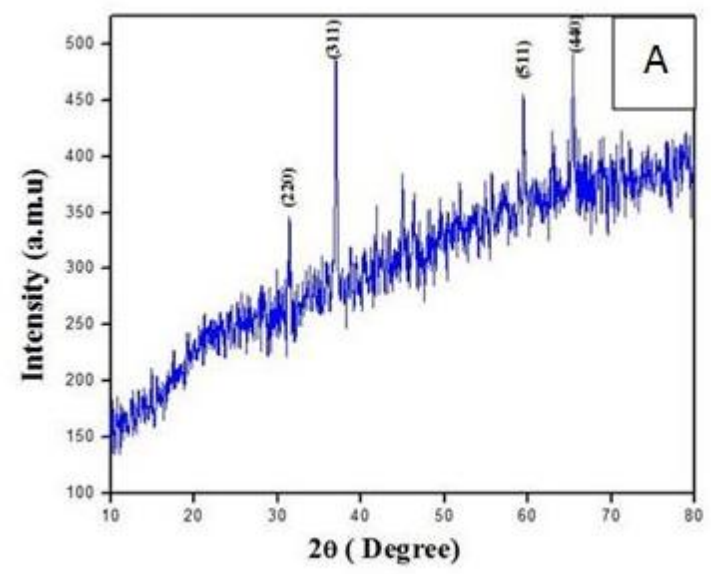

samples were identified by ATR spectroscopic studies. The recorded ATR spectra of the samples are shown in Figure 2. (A, B) The absorption peak at $643,651,531,539 \mathrm{~cm}^{-1}$ is due to the presence of cobalt oxide stretching and bending vibration [25]. The peak $1167,1180 \mathrm{~cm}^{-1}$ to the vibration modes of cadmium ions.

\subsection{UV Vis spectroscopy}

Figure 3. (A) (B) shows the UV-vis-NIR absorption spectrum. It is observed that absorption band maximum at $204 \mathrm{~nm}, 220 \mathrm{~nm}$ due to surface plasma resonances at the surface of the nanoparticle [26]. The absorption edge of the nanoparticles related to the size of the nanoparticles. quantum size effect become dominating when the size of the Nano crystallites is less then bulk excition bohr radius and it affects the electronic energy bands of the semiconductors [27].

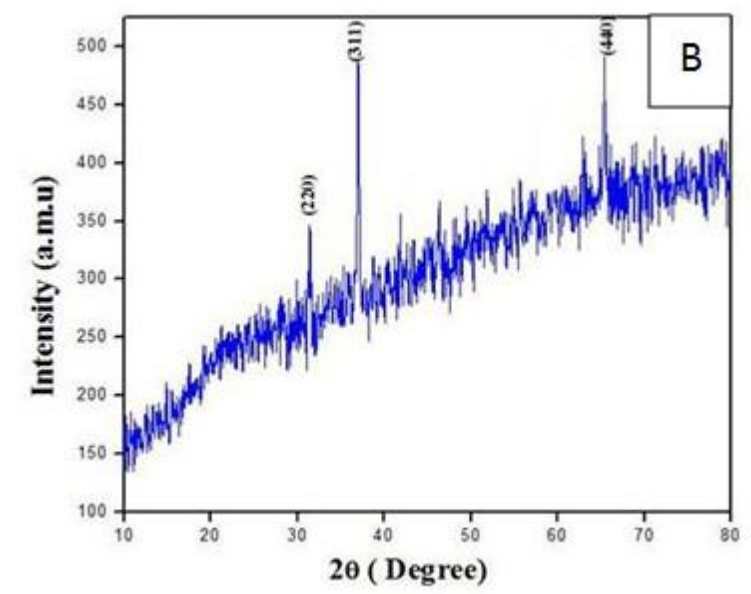

Figure 1 Powder X-ray diffraction pattern (A) without irradiation (B) with irradiation cadmium doped Co3O4 nanoparticles
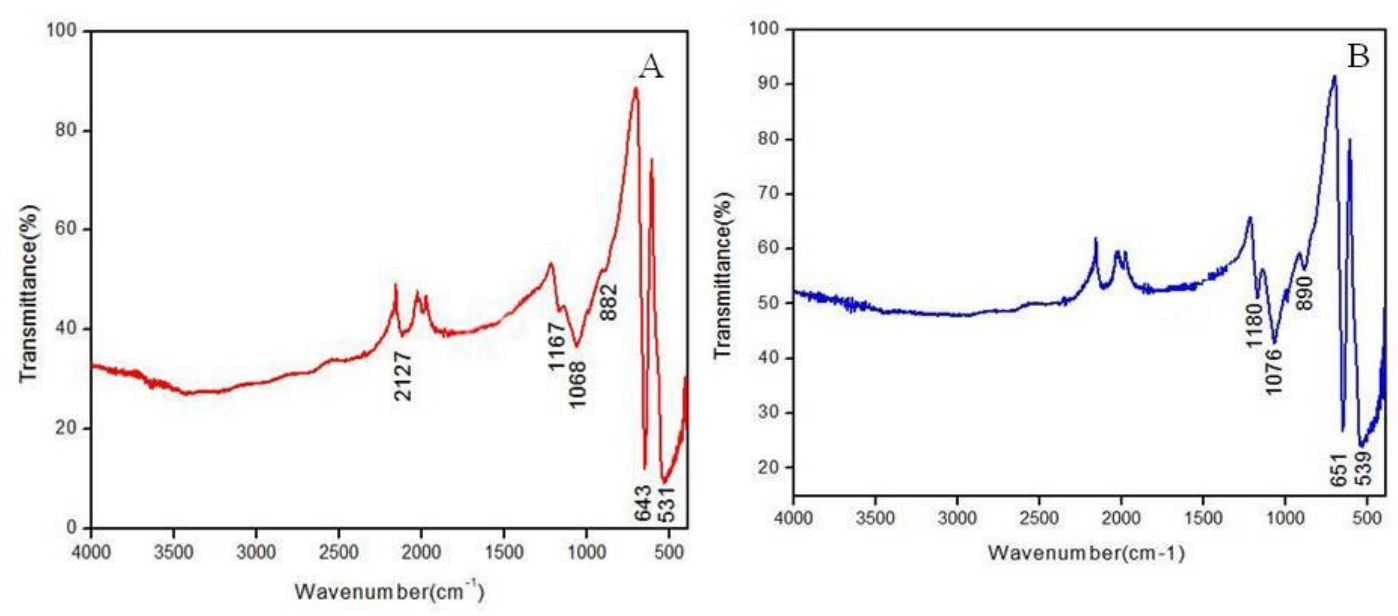

Figure 2. Shows the ATR Spectrum (A) without irradiation (B) with irradiation cadmium doped $\mathrm{Co}_{3} \mathrm{O}_{4}$ nanoparticles 

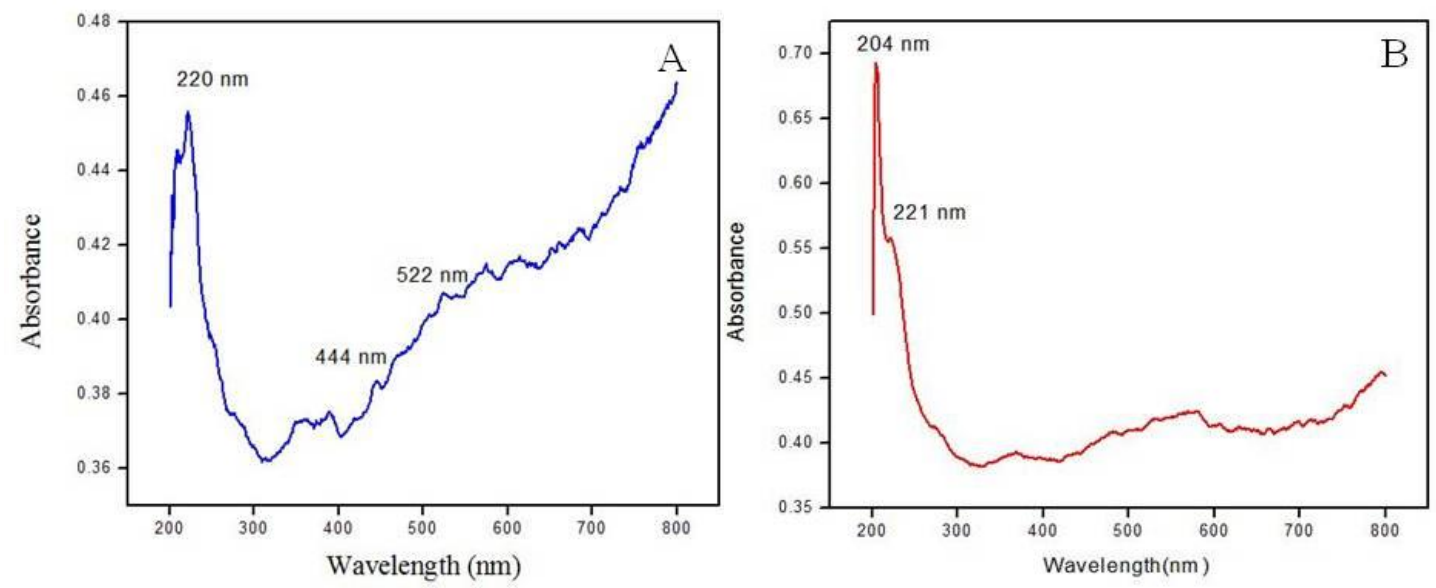

Figure 3. Shows the UV-Vis-NIR absorption spectrum (A) without irradiated (B) with irradiated cadmium doped $\mathrm{Co}_{3} \mathrm{O}_{4}$ nanoparticles
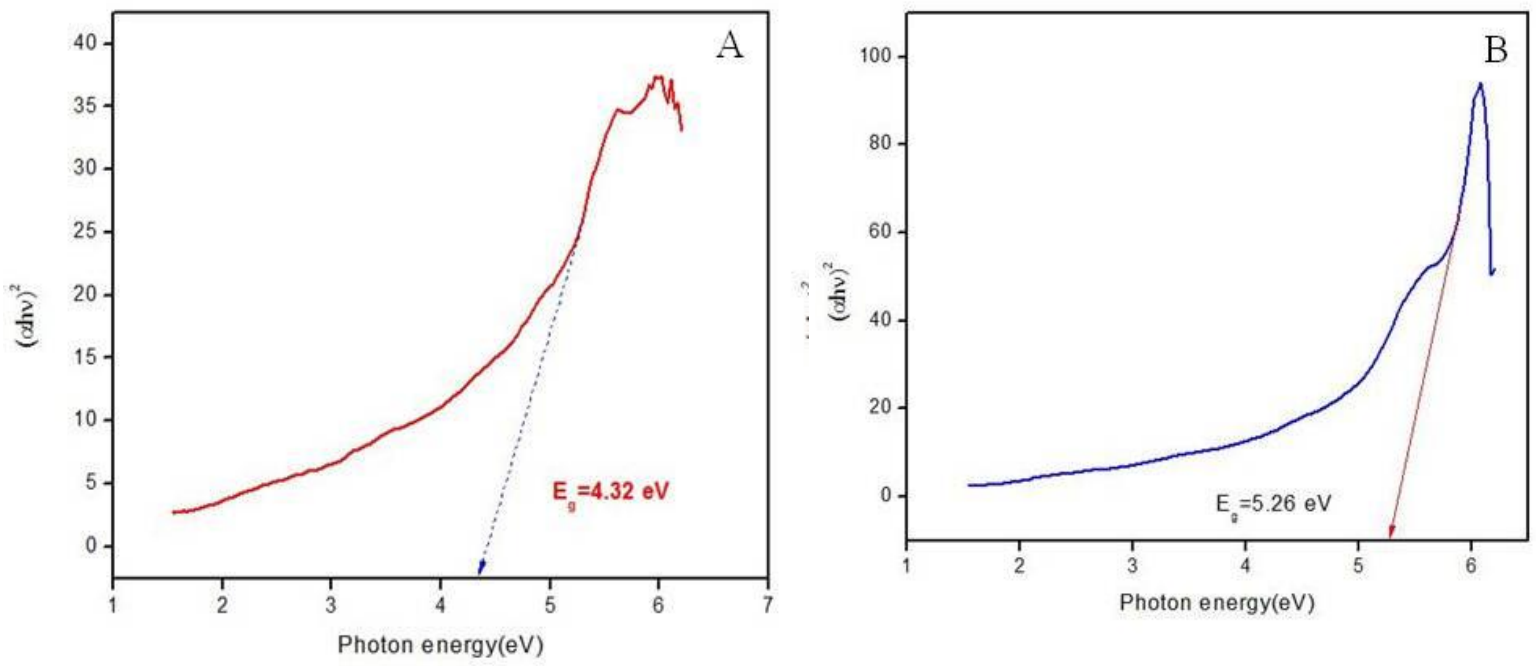

Figure 4. Shows the Indirect transition band gap shows plot photon energy (hv) eV verses (ahv)2(eV) (A)without irradiated $(\mathrm{B})$ with irradiated cadmium doped $\mathrm{Co}_{3} \mathrm{O}_{4}$ nanoparticles

\subsubsection{Determination of Energy Band Gap (Eg)}

Tauc and Davis-Mott relation following formula

$$
(\alpha h v)^{\mathrm{n}}=A(h v-\mathrm{Eg})
$$

Fig. 4. (A, B) Shows in Tauc plot method we plot energy on $\mathrm{X}$-axis while $(\mathrm{ahv})^{2}(\mathrm{eV})$ on the $\mathrm{y}$-axis. The cadmium sulphate doped cobalt oxide optical band gap values to be $E_{g}=4.32 \mathrm{eV}$ and $E_{g}=5.26 \mathrm{eV}$. The bandgap of material introduction of impurities, which create shallow states in the bandgap. shallow states have small ionization energies, and when the doping densits high, the dopent generate a band. Among the narrower bandgap for the absorption of the visible light [28].

\subsubsection{Determination of extinction co-efficient $(K)$}

The extinction coefficient $(K)$ explains the amount of absorption when electromagnetic wave propagates through a medium and it can be used following relation

$$
\mathrm{K}=\frac{\alpha \lambda}{4 \pi}
$$

Fig.5 (A, B) shows the extinction coefficient, versus wavelength for investigated sample. The extinction coefficient values increase with increasing photon energy.

\subsubsection{Determination of skin depth ( $\alpha$ )}

The skin depth $(\chi)$ was calculated using the following formula

$$
\chi=1 / \alpha
$$



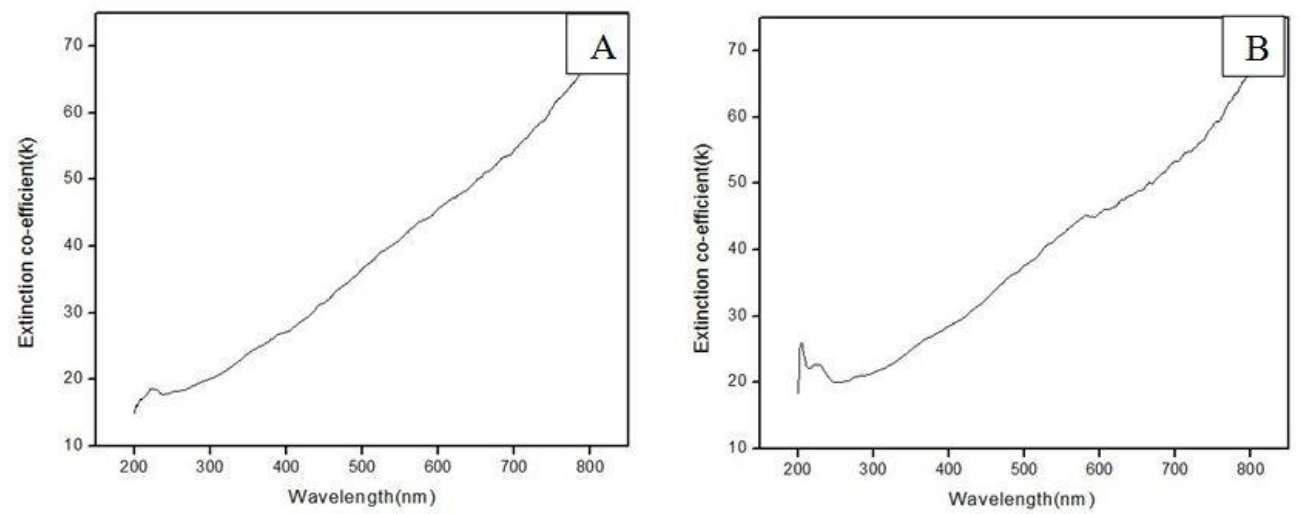

Figure 5 (A, B) Shows the Plot of wavelength versus extinction coefficient
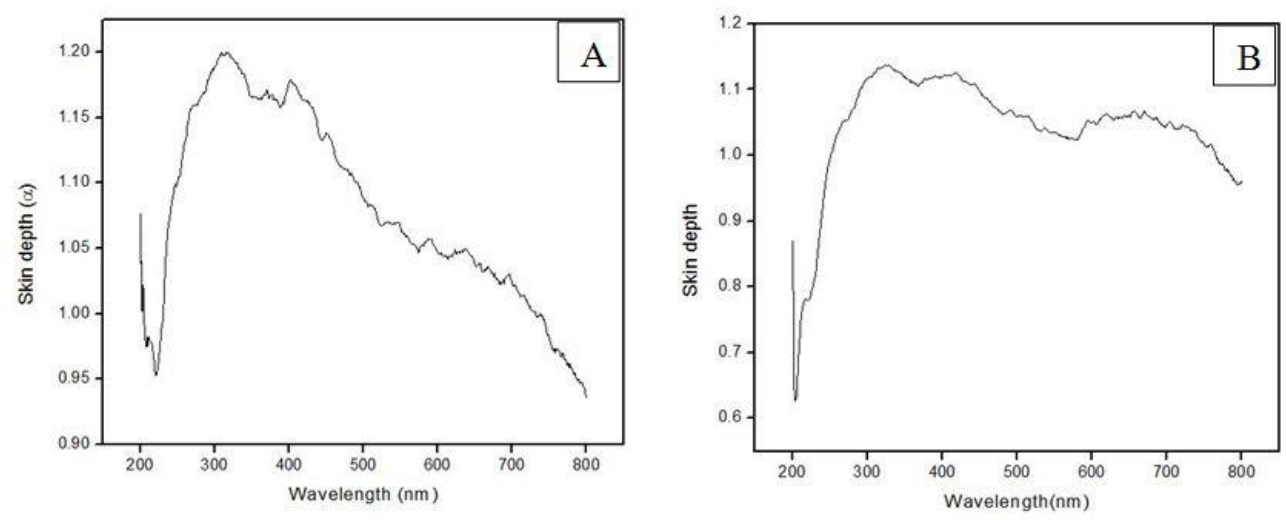

Figure.6 (A, B) Shows the Plot of wavelength versus skin depth
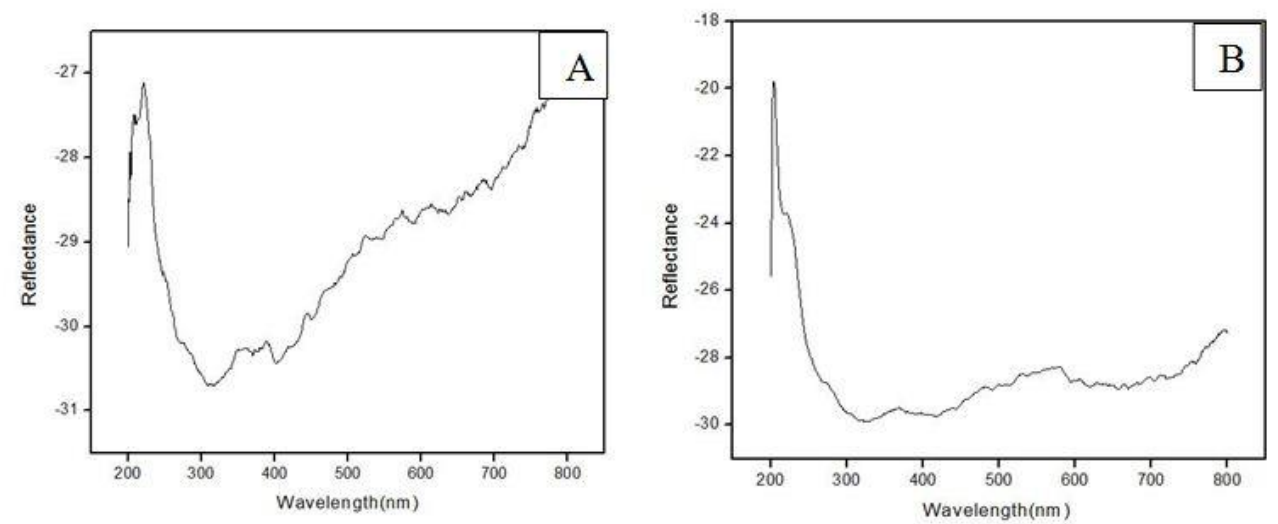

Figure. 7 (A, B) Shows the Plot of wavelength versus reflectance

Fig.6 (A, B) shows the skin depth, versus wavelength for investigated sample. Skin depth values decrease with increasing photon energy.

\subsubsection{Determination of reflectance $(R)$}

The Reflectance $(R)$ was calculated by using the relation

$$
\mathrm{R}=[1-\mathrm{T} \exp \mathrm{A}]^{1 / 2}
$$

Fig 7. (A, B) shows reflectance verses wavelength, and it's indicated that decreases reflectance with photon energy increases.

\subsubsection{Determination of refractive index (n)}

The Refractive index ( $n$ ) was calculated from the following formula 

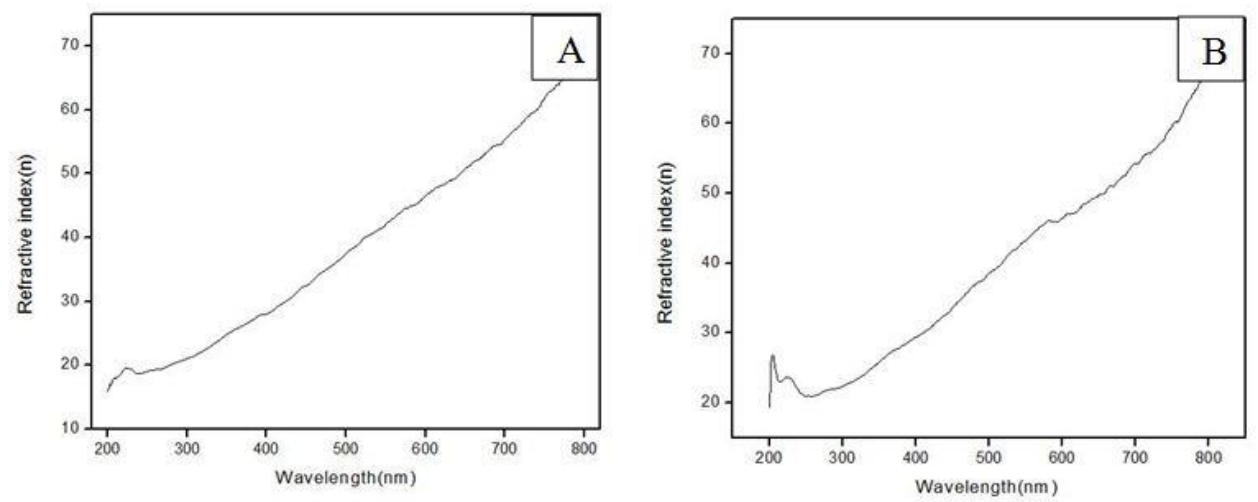

Figure. 8 (A, B) Shows the Plot of wavelength versus refractive index

$$
n=\left\{\left[\frac{1+R}{1-R}\right]^{2}-\left[1-K^{2}\right]\right\}^{1 / 2}-\left[\frac{1+R}{1-R}\right]
$$

Fig $8(A, B)$ shows the refractive index in the visible region for investigated sample. It is clear that the refractive index increases slowly with increasing the wavelength.

\section{Conclusion}

The cadmium doped cobalt oxide nanoparticles were synthesized by microwave irradiation method. The Powder $X$ ray diffraction confirms good crystalline nature. The FTIR-ATR confirms the presence of functional groups in the sample. UV-Vis spectroscopy results shows optical absorption bandgap of the cadmium doped cobalt oxide nanoparticles were estimated to be 4.32 and $5.26 \mathrm{eV}$, which are blue shifted in the absorption edge might be due to decrease in lattice strain. The optical parameter skin depth, extinction co-efficient, reflectance, refractive index is calculated and the prepared nanoparticles suitable for optical device applications.

\section{References}

[1] Xiaohe Liu, Guangzhou Qiu, and Xingguo Li Shape controlled synthesis and properties of uniform spinel cobalt oxide nano cubes, Nanotechnology, 16 (2005) 3035-3040. [DOI]

[2] L.M. Alrehaily, J.M. Joseph, M.C. Biesinger, D.A.Guzonas, J.C.Wren, Gamma-radiolysisassisted cobalt oxide nano particle formation RSC Publishing, Physical Chemistry Chemical Physics, 15 (2013) 1014-1024. [DOl]

[3] F. Moro, S. V. Y. Tang, F. Tuna, E. Lester, Magnetic properties of cobalt oxide nanoparticles synthesised by a continuous hydrothermal method, Journal of magnetism and magnetic materials, 348 (2013) 1-7. [DOl]

[4] S.G. Kandalkar, D.S. Dhawale, Chang-Koo Kim,
C.D. Lokhande, Chemical synthesis cobalt oxide thin filim electrode for super capacitor application, Synthetic metals, 160 (2010) 12991302. [DOI]

[5] S. R. Zhang, Z. B. Hu, K. Y. Liu, Y. Z. Liu, H. E. Fang, Q. L. Xie, Synthesis and characterization of porous cobalt oxide/copper oxide nanoplate as novel electrode material for supercapacitors, Transactions of Nonferrous Metals Society of China, 25 (2015) 4054 - 4062. [DOl]

[6] A. T. Khalil, M. Ovais, I. Ullah, M. Ali, Z. K. Shinwari, M. Maaza, Physical properties, biological applications and biocompatibility studies on biosynthesized single phase cobalt oxide $\left(\mathrm{Co}_{3} \mathrm{O}_{4}\right)$ nanoparticles via Sageretia thea (Osbeck.), Arabian Journal of Chemistry, 13 (2020) 606-619. [DOI]

[7] S. Chattopadhyay, S.P. Chakraborty, D. Latha, R. Baral, P. Pramanik, S. Roy, Surface modified cobalt oxide nano particles new opportunities for anti-cancer drug development, Cancer nanotechnology, 3 (2012) 13-23. [DOl]

[8] A. Ashok, A. Kumar, R. R. Bhosale, F. Almomani, M. A. H. S. Saad, S. Suslov, F. Tarlochan, Influence of fuel ratio on the performance of combustion synthesized bifunctional cobalt oxide catalysts for fuel cell application, International Journal of Hydrogen Energy, 44 (2019) 436-445. [DOl]

[9] C. Sun, X. Su, F. Xiao, C. Niu, J. Wang, Synthesis of nearly monodisperse Co3O4 nanocubes via a microwave-assisted solvothermal process and their gas sensing properties, Sensors and Actuators B: Chemical, 157 (2011) 681-685. [DOl]

[10] G. Patrinoiu, V. Etacheri, S. Somacescu, V. S. Teodorescu, R. Birjega, D. C. Culita, C. N, Hong, M. C. Morenoa, V.G.Pol, O. Carp, Spherical cobalt/cobalt oxide-Carbon composite anodes for enhanced lithium-ion storage, Electrochimica Acta, 264 (2018) 191-202. [DOI] 
[11] S. Gopinath, B. Karthikeyan, C. Ragupathi, K. Sivakumar, R. Sundaram, Structural, morphological, optical and magnetic properties of $\mathrm{Co}_{3} \mathrm{O}_{4}$ nanoparticles prepared by conventional method, Physica E: LowDimensional Systems and Nanostructures, 81 (2016) 66-70. [DOI]

[12] M. Durano, A.H. Tamboli, H. Kim, Cobalt oxide synthesized using urea precipitation method as catalyst for the hydrolysis of sodium borohydride, Colloids and Surfaces A: Physicochemical and Engineering Aspects, 520 (2007) 355-360. [DOl]

[13] S.M. Ansari, R.D. Bhor, K.R. Pai, D. Sen, S. Mazumder, Karthick Ghosh, Y.D. Kolekara, C.V. Ramana, Cobalt nano particles for biomedical applications: Facile synthesis, Physiochemical characterization, cytotoxicity behaviour and biocompatibility, Applied surface science, 414 (2017) 171-187. [DOl]

[14] G.A. Santos, C.M. Santos, S.W. da Silva, E.A. Urquieta-González, P.P.C. Sartoratto, Sol-gel synthesis of silica-cobalt composites by employing $\mathrm{CO}_{3} \mathrm{O}_{4}$ colloidal dispersions, Colloids and Surfaces A: Physicochemical and Engineering Aspects, 395 (2012) 217-224. [DOI]

[15] G. Wang, X. Shen, J. Horvat, B. Wang, H. Liu, D. Wexler, J. Yao, Hydrothermal synthesis and optical, magnetic, and supercapacitance properties of nanoporous cobalt oxide nanorods, The Journal of Physical Chemistry C, 113 (2009) 4357-4361. [DOI]

[16] S.K. Meher, G.R. Rao, Ultralayered $\mathrm{Co}_{3} \mathrm{O}_{4}$ for high-performance super capacitor applications, Journal of Physical Chemistry C, 115 (2011) 15646-15654. [DOI]

[17] J. Mu, L. Zhang, G. Zhao, Y. Wang, The crystal plane effect on the peroxidase-like catalytic properties of Co 3 O 4 nanomaterials, Physical Chemistry Chemical Physics, 16 (2014) 1570915716. [DOI]

[18] G. Allaedini, A. Muhammad, (2013) Study of influential factors in synthesis and characterization of cobalt oxide nanoparticles, Journal of Nanostructure in Chemistry, 3 (2013) 1-16. [DOI]

[19] S. Deng, X. Xiao, G. Chen, L. Wang, Y. Wang, $\mathrm{Cd}$ doped porous $\mathrm{Co}_{3} \mathrm{O}_{4}$ nanosheets as electrode material for high performance supercapacitor application, Electrochimica Acta, 196 (2016) 316-327. [DOI]

[20] R. Sathyamoorthy, P. Sudhagar, A. Balerna, C. Balasubramanian, S. Bellucci, A. I Popov, K. Asokan, Surfactant-assisted synthesis of Cd1xCoxS nanocluster alloys and their structural, optical and magnetic properties, Journal of Alloys and Compounds, 493 (2010) 40-245. [DOI]
[21] A.K Singh, U.T. Nakate, Microwave synthesis, characterization, and photoluminescence properties of nanocrystalline zirconia, The Scientific World Journal, 2014 (2014) 1-8. [DOI]

[22] V. chikan, E.J. McLaurin, Rapid nanoparticle synthesis by magnetic and microwave heating, Nanomaterials, 6 (2016) 1-9. [DOl]

[23] T. Kim, G. Jung, S.Yoo, K. S. Suh, R.S. Ruoff, (2013) Activated graphene-based carbons as supercapacitor electrodes with macro-and mesopores, ACS nano, 7 (2013) 6899-6905. [DOI]

[24] P. Nagaraju, A. Alsalme, A. Alswieleh, R. Jayavel, Facile in-situ microwave irradiation synthesis of $\mathrm{TiO}_{2} /$ graphene nanocomposite for high-performance supercapacitor applications, Journal of electro analytical chemistry, 808 (2018) 90-100. [DOI]

[25] S. Vijayakumar, A.K. Ponnalagi, S. Nagamuthu, G. Muralidharan, Microwave assisted synthesis of $\mathrm{Co} 3 \mathrm{O} 4$ nanoparticles for high-performance supercapacitors, Electrochimica Acta, 106 (2013) 500-505. [DOI]

[26] A. Lakehal, B. Bedhiaf, A. Bouaza, B. Hadj, A. Ammari, C. Dalache, Structural, optical and electrical properties of $\mathrm{Ni}$-doped $\mathrm{Co}_{3} \mathrm{O}_{4}$ prepared via Sol-Gel technique, Materials Research, 21 (2018). [DOl]

[27] S. Satheeshkumar, V. Jeevantham, D. Tamilselvi, Effect of Cu-doping on the structural and optical properties of $\mathrm{ZnO}$ nano crystallites prepared by co precipitation method, journal of ovonic research, 14 (2018) 9-15.

[28] S. Farhadi, J. Safabakhsh, P. Zaringhadam, (2013) Synthesis, characterization, and investigation of optical and magnetic properties of cobalt oxide $\left(\mathrm{CO}_{3} \mathrm{O}_{4}\right)$ nanoparticles, Journal of Nanostructure in Chemistry, 3 (2013) 1-9. [DOI]

\section{Acknowledgments}

The Authors are thankful to Alagappa university karaikudi, Karunya University Coimbatore, SAIF IIT Madras for characterization studies.

\section{Funding}

No funding was received for conducting this study.

\section{Conflict of interest}

The authors have no conflicts of interest to declare that they are relevant to the content of this article.

\section{About the License}

(C) The author(s) 2021. The text of this article is open access and licensed under a Creative Commons Attribution 4.0 International License 